\title{
Strain-designed strategy to induce and enhance second-harmonic generation in centrosymmetric and noncentrosymmetric materials
}

\author{
Eleonora Luppi \\ Sorbonne Universités, UPMC Univ Paris 06, UMR 7616, Laboratoire de Chimie Théorique, F-75005 Paris, France \\ and CNRS, UMR 7616, Laboratoire de Chimie Théorique, F-75005 Paris, France
}

Elena Degoli

Dipartimento di Scienze e Metodi dell'Ingegneria, Università di Modena e Reggio Emilia, Via Amendola 2 Padiglione Morselli, I-42122 Reggio Emilia, Italy and CNR-Istituto di Nanoscienze-S3, via Campi 213/A, I-41100 Modena, Italy

Matteo Bertocchi

Dipartimento di Scienze e Metodi dell'Ingegneria and Centro Interdipartimentale InterMech, Università di Modena e Reggio Emilia, Via Amendola 2 Padiglione Morselli, I-42122 Reggio Emilia, Italy

Stefano Ossicini

Dipartimento di Scienze e Metodi dell'Ingegneria and Centro Interdipartimentale En\&Tech, Università di Modena e Reggio Emilia, Via Amendola 2 Padiglione Morselli, I-42122 Reggio Emilia, Italy

and CNR-Istituto di Nanoscienze-S3, via Campi 213/A, I-41100 Modena, Italy

Valérie Véniard

Laboratoire des Solides Irradiés, Ecole Polytechnique, CNRS (UMR 7642), CEA-DSM-IRAMIS, Université Paris-Saclay, 91128 PALAISEAU cedex, France and European Theoretical Spectroscopy Facility (ETSF), France

(Received 12 March 2015; revised manuscript received 20 May 2015; published 13 August 2015)

\begin{abstract}
Second-harmonic generation is described by the second-order nonlinear susceptibility $\chi^{(2)}$ which, in the electric-dipole approximation, requires a noncentrosymmetric medium. It is very challenging and of high technological interest to search whether it is possible to find a way to break inversion symmetry in centrosymmetric crystals in order to induce second-order nonlinearities. A new intriguing way to observe second-order nonlinear phenomena is strain. Here, we present a detailed analysis of the correlation between the strain and the $\chi^{(2)}$ in both centrosymmetric and noncentrosymmetric materials. We considered $\mathrm{Si}$ and $\mathrm{SiC}$ as test materials and we studied different types of strain (tensile/compressive), in different directions (uniaxial/biaxial) and for different light-polarization directions. We found which is the type of strain necessary in order to induce, tune, and enhance second-harmonic generation in different energy regions for centrosymmetric and noncentrosymmetric materials.
\end{abstract}

DOI: 10.1103/PhysRevB.92.075204 PACS number(s): 71.15.Mb, 42.65.An, 42.65.Ky, 78.20.-e

\section{INTRODUCTION}

Second-harmonic generation (SHG) is a nonlinear optical process that converts two photons of frequency $\omega$ into a single photon of frequency $2 \omega$. SHG can be described by secondorder nonlinear susceptibility $\chi^{(2)}$ which, in the electric-dipole approximation, requires a noncentrosymmetric medium [1,2]. In fact, $\chi^{(2)}$ is zero in centrosymmetric systems because of the inversion symmetry and the first dipolar nonlinear optical processes possible are those related to third-order nonlinear susceptibility $\chi^{3}$ as, for example, the third-harmonic generation $[3,4]$ that converts three photons of frequency $\omega$ into a single photon of frequency $3 \omega$. However, third-order nonlinear effects are in principle much less efficient than second-order nonlinear effects [3]. Therefore, it is scientifically challenging and highly desirable for technological applications to find a way to break inversion symmetry in centrosymmetric crystals in order to induce significant second-order nonlinearities.

One of the most used approaches to measure secondorder response in centrosymmetric systems is to break the symmetry by cutting a surface $[5,6]$ or by interfacing two different centrosymmetric materials [7-9]. In both cases, only few atomic or molecular layers participate in the symmetry breaking and are at the origin of the SHG signal. That is why the SHG process has been largely used, through the years, as a highly selective surface/interface optical probe $[9,10]$.

Furthermore, in the last decade, second-order response has been largely used in the silicon photonics industry to search for new materials such as Si photonic-crystal nanocavities [11] and nanocrystals [12] for optoelectronic applications.

Very recently, this search for novel materials brought into evidence a new intriguing way of controlling second-order nonlinear phenomena: the strain. The strain is the structural deformation of a solid due to stress which is the pressure/tension exerted on a material by an external force. The strain can be induced in different layers of a material through the deposition of a second material with a different lattice constant. The thickness of such strained layers can vary within a wide range depending on the different experimental techniques and conditions used to deposit the lattice mismatching material.

The first investigations of the effects of strain on the second-harmonic signal in silicon date back to the late 1980s [13] where the inhomogeneous deformation of the crystal lattice at a strained interface layer was investigated in reflection. An increase in the second-harmonic signal by more 
than two orders of magnitude due to mechanical stress was reported by Govorkov et al. [13] for a $\mathrm{Si}_{x} \mathrm{Ni}_{y}$ polycrystalline straining layer. Recently, Schriever et al. [14] confirmed the predicted linear relationship by measuring the reflected SHG signal at (111) oriented thermally oxidized silicon interfaces in reflection, using a fundamental wavelength of $800 \mathrm{~nm}$. Jacobsen et al. [15] studied the optical response from photonic crystal waveguide where the periodically microstructured material surrounding the waveguide was made of silicon with holes that are partially filled with glass and air. These examples demonstrated a dependence between strain and SHG; however, the experimentally observed SHG signal contains, together with strain, also a component due to surface effect.

Very recently, the enhancement of SHG signal in bulk strained materials was experimentally observed by Cazzanelli et al. [16] in silicon waveguides stressed by silicon nitride. Various cladding layers of silicon nitride were used to change the applied stress and to induce the inhomogeneous strain needed as the source for the enhanced second-harmonic signal. They also measured the distribution of the strain inside the waveguide by a micro-Raman setup in a backscattering configuration, which allowed the shifts in the vibrational modes related to the strain state of the silicon to be detected. The optical properties of the strained silicon were then determined using a laser beam at a wavelength of $2.3 \mu \mathrm{m}(0.5 \mathrm{eV})$. We supported this experimental work by theoretical calculations studying deformed atomic structure of silicon lattice with ab initio methods. Similar results were also found by Chmielak et al. [17] on the investigation of local strain distribution and linear electro-optic effect in strained silicon waveguides. They have shown that the waveguide geometry has a strong influence on the induced strain asymmetry and on the resulting SHG signal in strained silicon waveguides. The presented results demonstrate that increasing the optical nonlinearity of strained silicon waveguides is possible by optimizing the geometry of the waveguides. It is worth noting that even if Chmielak et al. [17] and Cazzanelli et al. [16] agree about the observation of a relation between the $\chi^{(2)}$ and the strain, there is a quantitative inconsistency between their results. It has been proposed that this discrepancy is due to fundamental differences in the strained waveguides [17]. Moreover, also a contribution from the silicon/silicon nitride interface has to be considered [18]. Despite these differences, all the observations presented in literature [16,17,19-23] establish a correlation between the strain and the $\chi^{(2)}$. However, a clear quantitative understanding of this correlation is still missing, which makes its application controversial for the improvement of optoelectronic devices.

Motivated by our previous theoretical and experimental work [16], we present, here, a detailed theoretical study about the correlation between the strain and the $\chi^{(2)}$ for centrosymmetric ( $\mathrm{Si})$ and noncentrosymmetric ( $\mathrm{SiC}$ ) materials. The goal of comparing $\mathrm{Si}$ and $\mathrm{SiC}$ is to use inhomogeneous strain to alter the optical properties of a material as an alternative approach with respect to mixing different materials. The main advantage is that the material essentially stays the same, which means that all of the technology processes known from the semiconductor industry can be applied to strained silicon.
We start by presenting in Sec. II how we modeled compressive and tensile strain (uniaxial/biaxial) for the $\mathrm{Si}$ and $\mathrm{SiC}$ structures. In Sec. III for each $\mathrm{Si}$ and $\mathrm{SiC}$ strained structure we calculated the $\chi^{(2)}$ for a large frequency range and for different light-polarization directions in the case of uniaxial strain. In Sec. IV, we extended the analysis of the previous section in the case of biaxial strain. Through these comparative studies we were able to understand which is the type of strain that can induce, tune, and enhance SHG in both centrosymmetric and noncentrosymmetric materials.

\section{MODELING COMPRESSIVE AND TENSILE STRAIN}

We calculated ground-state and electronic properties of strained bulk Si and SiC with density-functional theory (DFT) in the local-density approximation with norm-conserving pseudopotentials [24] and plane-wave basis set with the ABINIT [25,26] software package. For convergence we used a Monkhorst-Pack $7 \times 7 \times 3 k$-point grid and an energy cutoff of $15 \mathrm{Ha}$ for $\mathrm{Si}$ and $20 \mathrm{Ha}$ for $\mathrm{SiC}$.

All strained bulk $\mathrm{Si}$ and $\mathrm{SiC}$ structures were obtained starting from a tetragonal $\left(a=b=c / 2\right.$ and $\left.\alpha=\beta=\gamma=90^{\circ}\right)$ unit cell of $8 \mathrm{Si}$ or $\mathrm{Si} / \mathrm{C}$ atoms in their bulk centrosymmetric positions with lattice parameters $a=10.178$ bohrs and $c=$ 20.356 bohrs for $\mathrm{Si}$, and $a=8.188$ bohrs and $c=16.376$ bohrs for $\mathrm{SiC}$.

In panel (a) of Fig. 1 we show the unstrained unit cell in the standard $x y z$ Cartesian reference $(\hat{i}, \hat{j}, \hat{k})$ and in the $X Y Z$ Cartesian reference $(\hat{I}, \hat{J}, \hat{K})$ which is obtained by a $45^{\circ}$ rotation of the $x y$ plane. The relation between the two Cartesian references is $\hat{I}=\frac{1}{\sqrt{2}} \hat{i}-\frac{1}{\sqrt{2}} \hat{j}, \hat{J}=\frac{1}{\sqrt{2}} \hat{i}+\frac{1}{\sqrt{2}} \hat{j}$, and $\hat{K}=\hat{k}$. We decided to work in the $X Y Z$ reference because we could construct a unit cell with fewer atoms. The lattice vectors are $\mathbf{a}_{1}=\left(a \frac{\sqrt{2}}{2}, 0,0\right), \mathbf{a}_{2}=\left(0, a \frac{\sqrt{2}}{2}, 0\right)$, and $\mathbf{a}_{3}=(0,0, c)$, and the unit cell is translationally repeated in the whole space by applying the vector $\mathbf{R}=n_{1} \mathbf{a}_{1}+n_{2} \mathbf{a}_{2}+n_{3} \mathbf{a}_{3}$, which is a linear combination through the integers $n_{1}, n_{2}$, and $n_{3}$ of the lattice vectors. In panel (b) we show the projection of the eight atoms on the $Y Z$ plane (red circle) and on the $X Y$ plane (red circle and black cross). In the case of the $X Y$ plane, we distinguished superposed atoms with a black cross.

We simulated compressive strain by constructing a bond length shorter than the bond length of the unstrained bulk and the tensile strain by constructing a longer bond length. In particular, to simulate uniaxial strain we only modified the coordinates of two atoms along the $z$ axis [see panel (c)] of Fig. 1. In order to create a continuous strain between the cell replica, a crucial point is to choose the lattice vectors coherently with the applied strain. We had to redefine the lattice vector along the $z$ axis as $\tilde{\mathbf{a}}_{3}=(0,0, \tilde{c})$ for each of the strained structures which preserve the boundary conditions in the repetition of the unit cell in order not to add any further strain. The strained unit cell is then translationally repeated by the new vector $\tilde{\mathbf{R}}=n_{1} \mathbf{a}_{\mathbf{1}}+n_{2} \mathbf{a}_{\mathbf{2}}+n_{3} \tilde{\mathbf{a}}_{\mathbf{3}}$.

Modifying two $z$-axis coordinates implies four different classes of uniaxally strained systems: compressive/tensile (CT), compressive/compressive (CC), tensile/tensile (TT), and tensile/compressive (TC). These classes are represented in the C-T plane of Fig. 2. The Si strained systems which belong 
(a)

$$
8 \text { atoms unit cell (3D) }
$$
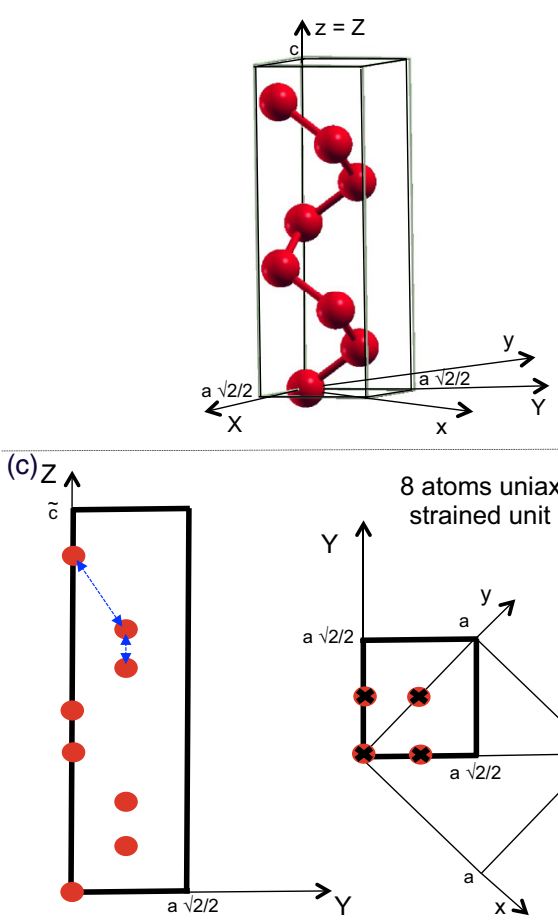

(b)

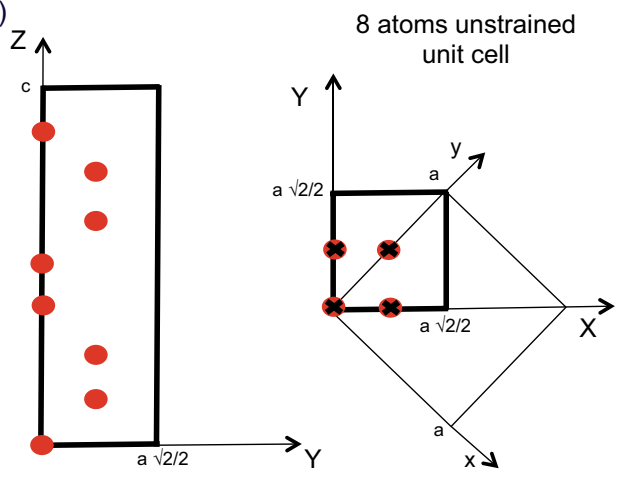

(d) $Z \uparrow$

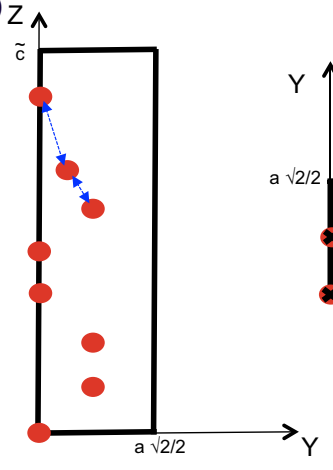

8 atoms biaxially strained unit cell strained unit cell

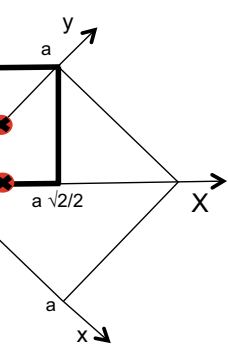

FIG. 1. (Color online) Panel (a) Unstrained eight atom unit cell in 3D panel. Panel (b) Unstrained eight atom unit cell projected in the $Y Z$ plane (red circle) and on the $X Y$ plane (red circle and black cross). Panel (c) Uniaxially strained eight atom unit cell. Panel (d) Biaxially strained eight atom unit cell.

to the first quadrant are CT, those belonging to the second quadrants are TT, those belonging to the third quadrant are $\mathrm{TC}$, and finally those belonging to the fourth quadrant are CC. It is worth noting that $\mathrm{T}>0$ corresponds to $\mathrm{C}<0$ and $\mathrm{C}>0$ corresponds to $\mathrm{T}<0$, as the tensile strain can be considered as a compressive strain with an inversion of the sign.

We classified each system by the percentage of variation of the strained bond length with respect to the unstrained bond. For example, a strained system called C0.7_T3.0 belongs to the class CT and it represents a system with the first bond compressed $0.7 \%$ and the second bond elongated $3.0 \%$.

Between all the uniaxial strained systems studied, we reported only 24 of them here. We believe that these systems cover a wide range of possible compressive and/or tensile strain and are sufficient to explain the trend with the $\chi^{(2)}$. In order to discuss this trend we analyzed our systems in four groups of six different strained structures. These groups are constructed keeping fixed one strain ( $\mathrm{C}$ or $\mathrm{T}$ ) and leaving the other strain (T or $\mathrm{C}$ ) free to change. The four groups are (1) C1.8_X: C1.8_C0.3, C1.8_C1.3, C1.8_C3.0, C1.8_T0.3, C1.8_T1.3, and C1.8_T3.0; (2) C0.7_X: C0.7_C0.3, C0.7_C1.3, C0.7_C3.0, C0.7_T0.3, C0.7_T1.3, and C0.7_T3.0; (3) T0.7_X: T0.7_T0.3, T0.7_T1.3, T0.7_T3.0, T0.7_C0.3, T0.7_C1.3, and T0.7_C3.0; (4) T1.8_X: T1.8_T0.3, T1.8_T1.3, T1.8_3, T1.8_C0.3, T1.8_C1.3, and T1.8_C3.0.

Moreover, the structures were classified by the stress tensor [27], i.e., the hydrostatic pressure (HP) (Fig. 2) which is equal to minus the average of the diagonal elements of the stress tensor and the shear stress (SS) which is related to the of-diagonal terms of the stress tensor. It is important to point out the different physical information which is given by HP, SS, and strain. In fact, the strain gives information about the local deformation of the structure, while HP and SS give information about the global deformation. Therefore, HP and SS take into account all the possible compensations between tensile and/or compressive local strain. For example, a structure which has zero or very low pressure can have a huge local strain. Moreover, a negative pressure means that the system is globally elongated even if locally it presents regions of compressive strain. Analogously, a positive pressure means that the system is globally compressed even if locally tensile strain can be present. It is then clear that, in order to describe the nature of the deformation in the materials, the knowledge of both strain and stress is necessary. The HP reported in Fig. 2 is calculated taking the HP of unstrained bulk as our reference.

In Fig. 2 we also plotted two bisectors which corresponds to $\mathrm{T}=\mathrm{C}$ (dashed-dotted red line) and $\mathrm{T}=-\mathrm{C}$ (dashed-dotted cyan line). The systems which lie on the bisector $\mathrm{T}=\mathrm{C}$ have the compressive strain exactly equilibrated by the tensile strain. Therefore, in this case HP is zero. Furthermore, the bisector $\mathrm{T}=\mathrm{C}$ separates the $\mathrm{C}-\mathrm{T}$ plane in two regions: an upper globally tensile region $(\mathrm{C}<\mathrm{T})$, where all systems show negative pressures and a lower globally compressive region $(\mathrm{C}>\mathrm{T})$, where all pressures are positive. The second bisector, which corresponds to $\mathrm{T}=-\mathrm{C}$, indicates those systems that, despite the applied strain, keep their centrosymmetric atomic 


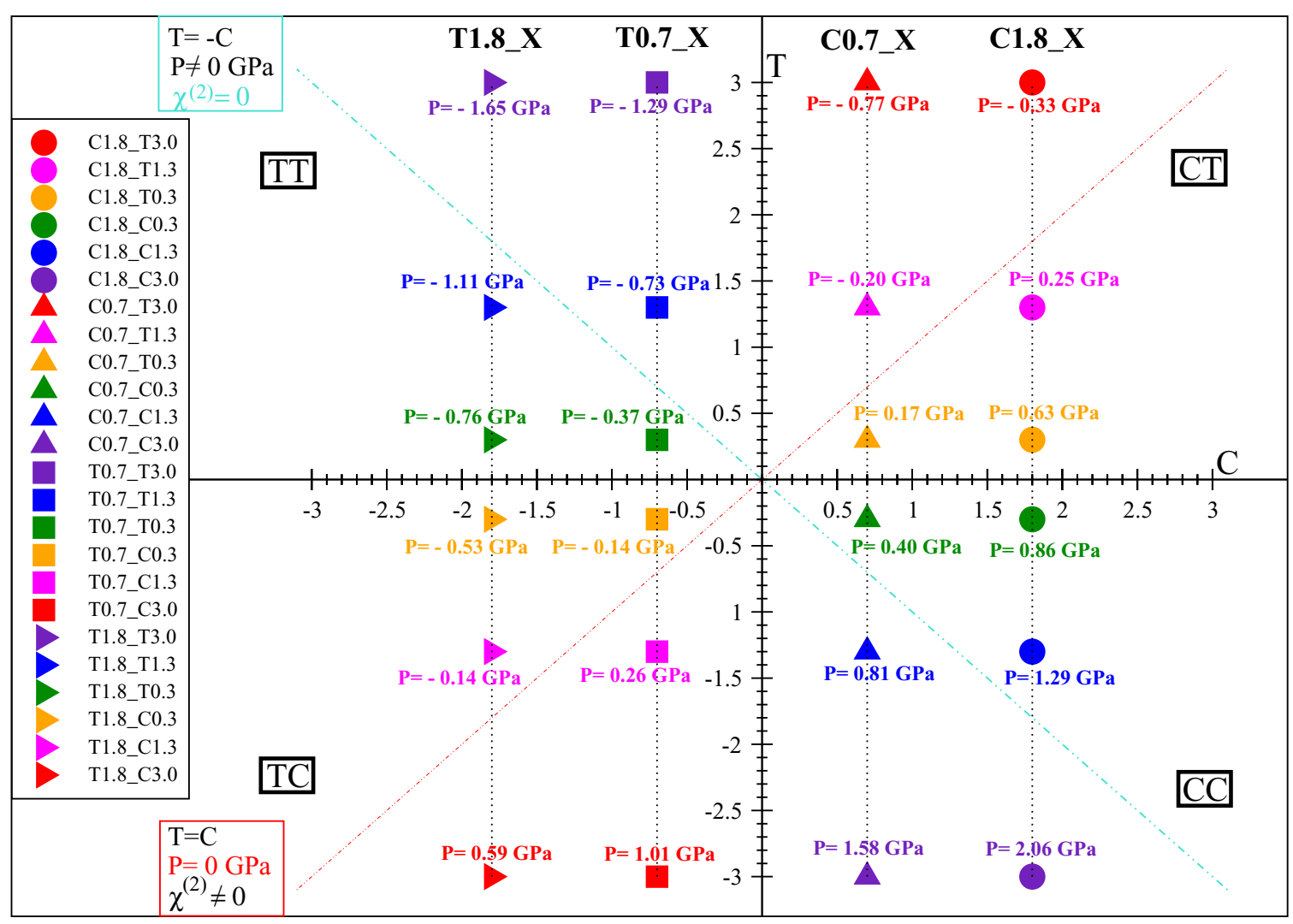

FIG. 2. (Color online) Representation of the 24 uniaxial strained structures studied on a C-T plane. The points on this plane are defined by the couple $(\mathrm{C}, \mathrm{T})$ and are correlated to the percentage of compression and elongation applied. For each structure the value of HP is reported. The bisector $\mathrm{T}=\mathrm{C}$ (dashed-dotted red line) and $\mathrm{T}=-\mathrm{C}$ (dashed-dotted cyan line) are also represented.

structure (i.e., $\chi^{(2)}=0$ ) [1]. In these cases the HP is usually quite high as no compensation effects between compressive and tensile strain occurs.

To clarify the notation we would like to note that in the group C1.8_X all the structures have a compressive bond of $1.8 \%(C=1.8 \%)$. In the case where also the second bond is compressed we have the fully compressive (CC) systems: C1.8_C0.3 $(\mathrm{C}=0.3 \%, \mathrm{HP}=0.86 \mathrm{GPa}), \mathrm{C} 1.8 \_\mathrm{C} 1.3(\mathrm{C}=$ $1.3 \%, \mathrm{HP}=1.29 \mathrm{GPa})$, and $\mathrm{C} 1.8 \_\mathrm{C} 3.0(\mathrm{C}=3.0 \%, \mathrm{HP}=$ $2.06 \mathrm{GPa})$. For these systems the local strain increases together with the stress (HP). Instead, in the case where the second bond is elongated we have systems of the type CT: C1.8_T0.3 $(\mathrm{T}=0.3 \%, \mathrm{HP}=0.63 \mathrm{GPa}), \mathrm{C} 1.8 \_\mathrm{T} 1.3(\mathrm{~T}=1.3 \%, \mathrm{HP}=$ $0.25 \mathrm{GPa})$, and C1.8_T3.0 $(\mathrm{T}=3.0 \%, \mathrm{HP}=-0.33 \mathrm{GPa})$. For these systems the local strain increases while the global stress (HP) diminishes. The opposite behavior of the stress towards strain is due to a compensation effect between compressive and tensile strain in the CT systems, which is not present in the CC systems. Moreover, increasing the percentage of elongation in these structures induces a transition from systems that are globally compressed ( $\mathrm{HP}>0$ ) to systems that are globally tensile $(\mathrm{HP}<0)$ such as, for example, in C1.8_T3.0. The systems which belong to the $\mathbf{C 0 . 7} \mathbf{X}$ group have a compressive bond of $0.7 \%(C=0.7 \%)$ and show the same trend for HP as the C1.8_X group. However, on average, the HP is lower as the compressive strain of $0.7 \%$ is lower than $1.8 \%$. The groups T0.7_X and T1.8_X are composed of three structures of fully tensile systems of the type TT and of three systems of type
TC. In the case of T0.7_X the first bond is elongated $0.7 \%$ ( $\mathrm{T}=0.7 \%$ ), while for $\mathbf{T 1 . 8} \_\mathbf{X}$ the same bond is elongated $1.8 \%(\mathrm{~T}=1.8 \%)$. We observed that, also in this case, the HP becomes greater with the increase of tensile strain and that in the case of compressive and tensile strain compensation effects are present.

Considering the biaxial strain, we modified the coordinates of two atoms along the $z$ axis and of just one atom along the $Y$ direction in the $x y$ plane [see panel (d) of Fig. 1].

We would also like to note that in order to preserve the geometrical strain in the structures, we did not relax uniaxially and biaxially strained $\mathrm{Si}$ and $\mathrm{SiC}$ cells.

In the following, we present the second-order susceptibility $\chi^{(2)}$ calculated in time-dependent density-functional theory (TDDFT) using the 2light code where our nonlinear TDDFT formalism is implemented [28,29]. We show $\chi^{(2)}$ in the independent-particle approximation (IPA) as we verified that the contribution from crystal-local fields and excitons is around $5 \%$ and it negligibly influences the trend with stress/strain. In fact, the local field effects slightly reduce the overall SHG intensity without modifying the shape of the spectrum, while the excitonic effects [28] have the opposite behavior. As this is the same for all the spectra, and the shape is substantially unchanged with respect to IPA, our results have more general validity.

A scissor operator of $0.6 \mathrm{eV}$ for $\mathrm{Si}$ and $0.8 \mathrm{eV}$ for $\mathrm{SiC}$ was used to simulate the quasiparticle energies. These values are used as they reproduce correctly the GW quasiparticle 

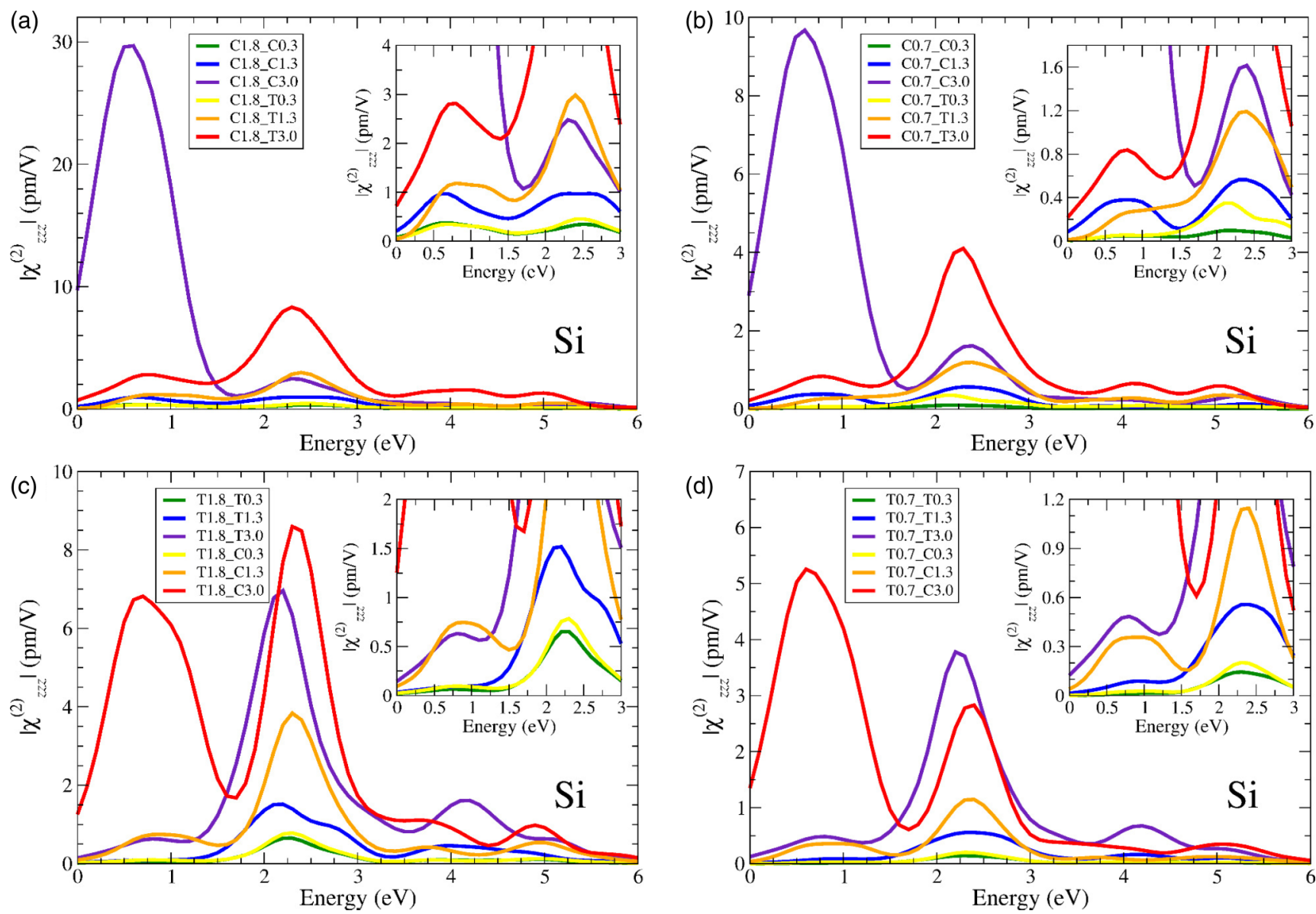

FIG. 3. (Color online) $\chi_{z z z}^{(2)}(\omega)$ spectra for uniaxial C1.8_X strained Si [panel (a)], uniaxial C0.7_X strained Si [panel (b)], uniaxial T1.8_X strained Si [panel (c)] and uniaxial T0.7_X strained Si [panel (d)]. The insets show a magnification of the low-energy regions.

band structure [29,30]. To converge optical spectra we used 5000 plane waves and 130 bands. In order to obtain faster convergence with respect to $K$ points we sampled the Brillouin zone using a mesh of irregular (random) $K$ points. We have tested and verified that, once convergence is achieved, random meshes and regular grids give the same results. For these calculations we used between 30000 and 40000 random $K$ points depending on the structures. Random meshes help us speed up convergence and reduce computational time. Indeed in our code, to increase the set of $K$ points where the Brillouin zone is sampled, in the case of regular grids we need to define a new finer grid and compute the wave functions and eigenvalues on all the points of the new grid. If we use random points we can always add the new points to the old set, solving the DFT self-consistent calculation on the new points only. Irregular meshes allow one to randomly access regions of the Brillouin zone that a regular mesh can access only if its step is small enough. Because of this, in general we observed that using irregular grids the number of points necessary to converge is usually smaller than in the case of regular grids. Finally, a Gaussian broadening of $0.013 \mathrm{eV}$ has also been applied.

\section{UNIAXIAL STRAIN AND $\chi^{(2)}$}

In Fig. 3 we show $\chi_{z z z}^{(2)}$ for $\mathbf{C 1 . 8 \_ X}[$ panel (a)], for C0.7_X [panel (b)], for T1.8_X [panel (c)], and for T0.7_X [panel (d)]. $\chi_{z z z}^{(2)}$ is also the only component different from zero for these systems. We observe that an important common feature of all the spectra is the presence of three different peaks which are almost at the same energy region. The first peak (I) is in the infrared region around $0.5-0.7 \mathrm{eV}$, the second peak (II) is in the visible region around $2.0-2.2 \mathrm{eV}$, and finally the third peak (III) is still in the visible region but around 3.8-4.0 eV. The transitions involved in peaks II and III can be identified by comparing with the absorption spectrum of Si [29]. In fact, peak III corresponds to the absorption peak in bulk silicon, while II is located precisely at half this energy. Looking at the mathematical expression of the $\chi^{(2)}$ [31] it is clear that the SHG spectrum always presents structures located at $\omega=\omega_{m n}$ and $\omega=\omega_{m n} / 2$ where $\omega_{m n}$ are the excitation energies of the material. Instead, peak I results from transitions in the gap of the material due to the strain. This structure can also be observed in absorption spectra (not shown here).

The absolute and relative intensities of the peaks can be very different between the spectra. The observed differences in the intensities are due to the different strain and stress between the structures. In order to better investigate the link between strain/stress and the $\chi_{z z z}^{(2)}$ we reported in Table I the value of $\chi_{z z z}^{(2)}(\mathrm{pm} / \mathrm{V})$ for the three main peaks (I, II, and III) (see also the Appendix). As a first remark we observed that the absolute intensity of the peaks increases going from smaller to larger strain. In fact, for the TT and CT systems belonging 
TABLE I. Maximum intensity (in pm/V) of the three main peaks: I, II, and III of the calculated $\chi_{z z z}^{(2)}$ for the systems represented in Fig. 2.

\begin{tabular}{|c|c|c|c|c|c|c|c|c|c|c|c|c|}
\hline & \multicolumn{6}{|c|}{ TT } & \multicolumn{6}{|c|}{ CT } \\
\hline & \multicolumn{3}{|c|}{$\begin{array}{c}\text { T1.8_X } \\
\mathrm{T}=1.8 \%\end{array}$} & \multicolumn{3}{|c|}{$\begin{array}{c}\text { T0.7_X } \\
\mathrm{T}=0.7 \%\end{array}$} & \multicolumn{3}{|c|}{$\begin{array}{c}\text { C0.7_X } \\
\mathrm{C}=0.7 \%\end{array}$} & \multicolumn{3}{|c|}{$\begin{array}{c}\text { C1.8_X } \\
\mathrm{C}=1.8 \%\end{array}$} \\
\hline & I & II & III & I & II & III & I & II & III & I & II & III \\
\hline $\mathrm{T}=3.0 \%$ & 0.63 & 6.96 & 1.61 & 0.48 & 3.78 & 0.68 & 0.84 & 4.10 & 0.65 & 2.89 & 8.21 & 1.49 \\
\hline $\mathrm{T}=1.3 \%$ & 0.09 & 1.52 & 0.46 & 0.09 & 0.56 & 0.16 & 0.28 & 1.19 & 0.28 & 1.18 & 2.99 & 0.40 \\
\hline \multirow[t]{5}{*}{$\mathrm{T}=0.3 \%$} & 0.06 & 0.65 & 0.10 & 0.01 & 0.14 & 0.03 & 0.06 & 0.35 & 0.09 & 0.35 & 0.46 & 0.07 \\
\hline & \multicolumn{6}{|c|}{ TC } & \multicolumn{6}{|c|}{$\mathrm{CC}$} \\
\hline & \multirow{2}{*}{\multicolumn{3}{|c|}{$\begin{array}{c}\text { T1.8_X } \\
T=1.8 \%\end{array}$}} & \multirow{2}{*}{\multicolumn{3}{|c|}{$\begin{array}{c}\text { T0.7_X } \\
\mathrm{T}=0.7 \%\end{array}$}} & \multirow{2}{*}{\multicolumn{3}{|c|}{$\begin{array}{c}\text { C0.7_X } \\
\mathrm{C}=0.7 \%\end{array}$}} & \multirow{2}{*}{\multicolumn{3}{|c|}{$\begin{array}{c}\text { C1.8_X } \\
\mathrm{C}=1.8 \%\end{array}$}} \\
\hline & & & & & & & & & & & & \\
\hline & I & II & III & I & II & III & I & II & III & I & II & III \\
\hline $\mathrm{C}=0.3 \%$ & 0.10 & 0.78 & 0.10 & 0.03 & 0.20 & 0.02 & 0.05 & 0.10 & 0.07 & 0.38 & 0.35 & 0.05 \\
\hline $\mathrm{C}=1.3 \%$ & 0.75 & 3.84 & 0.42 & 0.36 & 1.15 & 0.11 & 0.38 & 0.57 & 0.08 & 0.97 & 0.97 & 0.14 \\
\hline $\mathrm{C}=3.0 \%$ & 6.82 & 8.60 & 1.11 & 5.25 & 2.83 & 0.35 & 9.67 & 1.62 & 0.22 & 29.68 & 2.48 & 0.47 \\
\hline
\end{tabular}

to the classes T1.8_X, T0.7_X, C0.7_X, and C1.8_X, the absolute value of the peaks increases going from $\mathrm{T}=0.3 \%$ to $\mathrm{T}=1.3 \%$ and $\mathrm{T}=3.0 \%$. The same is observed for the $\mathrm{TC}$ and $\mathrm{CC}$ belonging to the same classes. In this case the absolute value of the peaks increases going from $\mathrm{C}=0.3 \%$ to $\mathrm{C}=1.3 \%$ and $\mathrm{C}=3.0 \%$.

For example, if we consider the first peaks of the $\chi_{z z z}^{(2)}$ for the $\mathrm{CC}$ systems belonging to the $\mathbf{C 1 . 8} \mathbf{X}$ class we observe that their values are $0.38 \mathrm{pm} / \mathrm{V}(\mathrm{C}=0.3 \%), 0.97 \mathrm{pm} / \mathrm{V}(\mathrm{C}=$ $1.3 \%)$, and $29.68 \mathrm{pm} / \mathrm{V}(\mathrm{C}=3.0 \%)$ being this last one also the strongest compressive structure. The second peak changes from $0.35 \mathrm{pm} / \mathrm{V}(\mathrm{C}=0.3 \%)$, to $0.97 \mathrm{pm} / \mathrm{V}(\mathrm{C}=1.3 \%)$ and to $2.48 \mathrm{pm} / \mathrm{V}(\mathrm{C}=3.0 \%)$. Finally, the third peak grows from $0.05 \mathrm{pm} / \mathrm{V}(\mathrm{C}=0.3 \%)$, to $0.14 \mathrm{pm} / \mathrm{V}(\mathrm{C}=1.3 \%)$ and finally to $0.47 \mathrm{pm} / \mathrm{V}(\mathrm{C}=3.0 \%)$. We observe that it is the first peak, in the infrared region, that is largely increased by the compressive strain.

Moving to the CT systems belonging to the $\mathbf{C 1 . 8} \mathbf{X}$ class one obtains for the first peak $0.35 \mathrm{pm} / \mathrm{V}(\mathrm{T}=0.3 \%)$, $1.18 \mathrm{pm} / \mathrm{V}(\mathrm{T}=1.3 \%)$, and $2.89 \mathrm{pm} / \mathrm{V}(\mathrm{T}=3.0 \%)$. For the second peak we have $0.46 \mathrm{pm} / \mathrm{V}(\mathrm{T}=0.3 \%), 2.99 \mathrm{pm} / \mathrm{V}$ $(\mathrm{T}=1.3 \%)$, and $8.21 \mathrm{pm} / \mathrm{V}(\mathrm{T}=3.0 \%)$ and finally for the third peak $0.07 \mathrm{pm} / \mathrm{V}(\mathrm{T}=0.3 \%), 0.40 \mathrm{pm} / \mathrm{V}(\mathrm{T}=1.3 \%)$, and $1.49 \mathrm{pm} / \mathrm{V}(\mathrm{T}=3.0 \%)$.

It is interesting to note that the presence of an elongation results in an enhancement of the second peak in the visible region around $2.5 \mathrm{eV}$ [see panel (a) of Fig. 3]. However, we never observe a peak as strong as the one we found in the case of CC. In the CT case, the compressive strain is compensated by the tensile strain and this affects the global pressure of the systems which is lower than those calculated in the $\mathrm{CC}$ for the same percentage of strain. These trends are confirmed for the C0.7_X and C1.8_X classes. It is still the second peak that is mainly enhanced by the presence of the tensile strain.

A similar result can be obtained for the T0.7_X and T1.8_X classes. We still observed that the intensity of $\chi_{z z z}^{(2)}$ increases with the increase of the local strain. The compressive strain still mainly influences the energy region below $1 \mathrm{eV}$, while the tensile greatly enhances the $\chi_{z z z}^{(2)}$ around $2.5 \mathrm{eV}$. Noteworthy for these systems, the intensity of the second peak dominates over the first one because of the tensile strain.

Comparing our findings with the recently experimentally measured $\chi^{(2)}$ in Si photonic crystal nanocavities [11] we observed that these values are much smaller than ours. Instead, on average, our $\chi^{(2)}$ intensities were in agreement with the experimental results on strained $\mathrm{Si}$ waveguides [16,17].

In this analysis we also noticed two special cases which correspond to the two bisectors in the C-T plane (see Fig. 2). In fact, in the region close to the bisector $\mathrm{C}=\mathrm{T}$ the HP is close or equal to zero; actually the compression is equilibrated by the elongation, but the local strain can be very high.

The calculated $\chi_{z z z}^{(2)}$ can be very intense with peaks comparable to that of systems subjected to almost the same amount of global strain. Instead, in the region onto the second bisector $\mathrm{T}=-\mathrm{C}$ the $\chi_{z z z}^{(2)}$ is equal to zero because the system is still centrosymmetric even if both the HP and the local strain can be very high.

For the uniaxial strain we can finally conclude that it is the mixing between compression and elongation that modulates the $\chi_{z z z}^{(2)}$ spectra. Taking the pressure as a parameter, it is evident that lower HP implies a weaker SHG signal. However, as the pressure gives only information about the global behavior of the system it is necessary to analyze the local strain in order to be able to understand and control the SHG. It is the relative weight of local tensile and compressive strain in the system that can permit one to tune the intensity at different frequency regions. In particular, it is important to underline that a compressive strain can induce a huge SHG in the infrared region (telecom wavelengths), while a tensile strain can enhance SHG in the visible region. Moreover, a compression will always induce stronger SHG than an elongation.

Instead, what happens if we strain a noncentrosymmetric system such as $\mathrm{SiC}$ ? To answer this question we calculated $\chi^{(2)}$ for strained $\mathrm{SiC}$ in the configurations C1.8_3.0, C1.8_T3.0, T1.8_T3.0, and T1.8_C3.0 and the results are shown in Fig. 4. Here, we considered as the reference the $\chi_{x y z}^{(2)}$ for unstrained $\mathrm{SiC}$ bulk. In fact, as unstrained $\mathrm{SiC}$ has zinc-blende symmetry the only component different from zero is the $\chi_{x y z}^{(2)}$. 

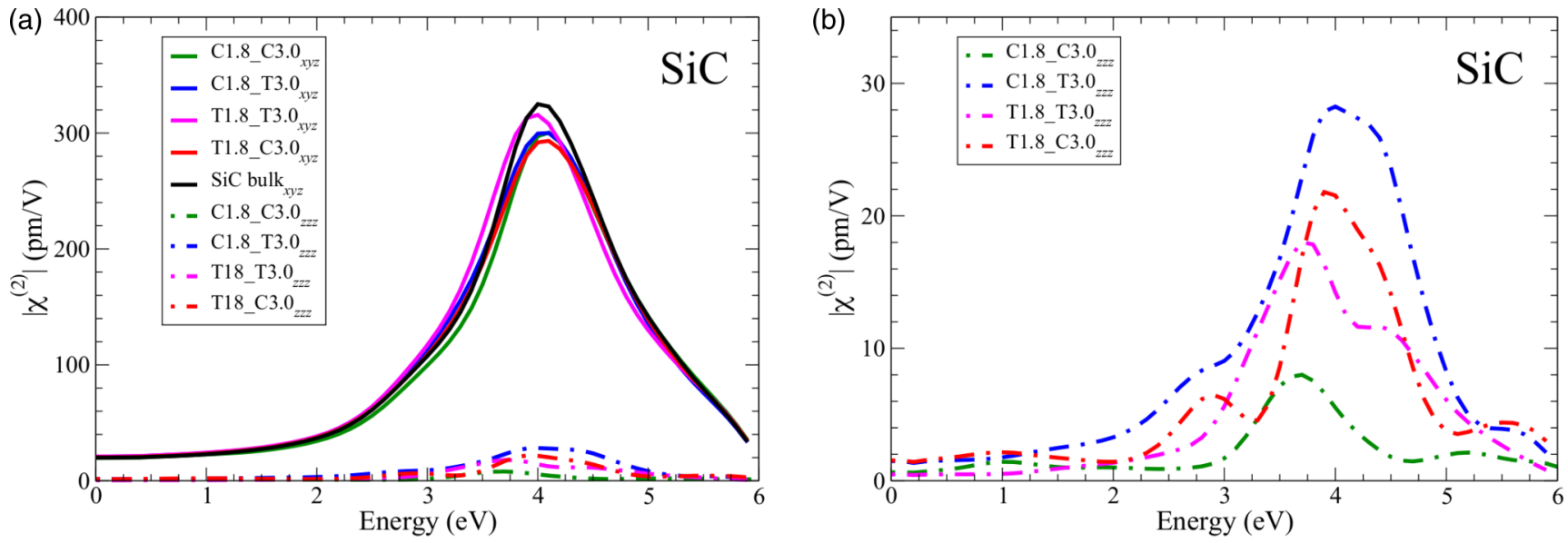

FIG. 4. (Color online) $\chi_{x y z}^{(2)}$ and $\chi_{z z z}^{(2)}$ spectra for unstrained bulk SiC and for $\mathbf{C 1 . 8} \_\mathbf{X}$ and T1.8_X strained SiC.

In panel (a) of Fig. 4 we show the $\chi_{z z z}^{(2)}$ and the $\chi_{x y z}^{(2)}$ spectra as they are the only nonzero components in strained $\mathrm{Si}$. We observed that for $\chi_{x y z}^{(2)}$ a uniaxial compressive/tensile strain does not significantly change the spectra with respect to the unstrained bulk value. Instead, the $\chi_{z z z}^{(2)}$ is now nonvanishing with the main peak that can increase up to $30 \mathrm{pm} / \mathrm{V}$. This magnitude is in the same range of the widely used $\mathrm{LiNbO}_{3}$. In particular, the peaks of the $\chi_{z z z}^{(2)}$ which are enhanced by the strain are observed between 3 and $5 \mathrm{eV}$ which corresponds to the energy region of the main peak of the spectrum for the unstrained $\mathrm{SiC}$ [see panel (b) of Fig. 4]. Anyway a small peak is still present in the region around $1 \mathrm{eV}$. We see that the CT combination (the one with greater strain gradient and therefore "greater" symmetry breaking) is the most effective and therefore CT systems provide the most intense spectra with respect to the pure $\mathrm{CC}$ and TT systems. Comparing the C1.8_T3.0 and T1.8_C3.0 spectra we note a trend similar to that in Si also if not so evident as there: actually the C1.8_T3.0 structure, which is globally tensile, has, between the two, the more intense peak in the visible, while the T1.8_C3.0, which is globally compressed, has the stronger peak in the infrared. Things go in parallel for the pure compressive (C1.8_C3.0) and pure tensile (T1.8_T3.0) structures: the spectrum of the tensile one is more intense in the visible, while the $\mathrm{CC}$ dominates in the infrared.

\section{BIAXIAL STRAIN AND $\chi^{(2)}$}

In order to better simulate possible experimental scenarios, we studied the role of biaxial compressive/tensile strain by changing the atomic coordinates in the $x y$ plane too. We labeled the biaxial structures using the same notation for the uniaxial structures, but we added a "Y" in the end of the name. For example, the C1.8_T3.0_Y corresponds to the uniaxial C1.8_T3.0 with an additional strain along the $Y$ direction.

We reported in Table II the pressure HP and shear stress $\mathrm{SS}_{Y Z}$ for $\mathrm{Si}$ and $\mathrm{SiC}$ in the case of the uniaxially strained C1.8_T3.0 and T1.8_C3.0 and of the biaxially strained C1.8_T3.0_Y and T1.8_C3.0_Y. We observe that the "Y" strain has a tensile effect (HP is diminished with respect to the corresponding uniaxial system) and a nonzero $\mathrm{SS}_{Y Z}$.
In Fig. 5 we show $\chi_{z z z}^{(2)}$ and $\chi_{Y Y Y}^{(2)}$ for the biaxially strained $\mathrm{Si}$ and $\mathrm{SiC}$. For comparison we reported also the $\chi_{z z z}^{(2)}$ for the uniaxially strained systems. In the case of Si [panel (a)] we observed that, starting from the uniaxial C1.8_T3.0 (dotdashed blue) and T1.8_C3.0 (dot-dashed yellow), the biaxial strain increases the $\chi^{(2)}$ intensity in particular below $1 \mathrm{eV}$ (infrared region) and the more enhanced component is the $\chi_{Y Y Y}^{(2)}$. In the case of SiC [panel (b)] we observe that the biaxial strain has less impact than in $\mathrm{Si}$ and for the component $\chi_{Y Y Y}^{(2)}$ we observe an overall increase at low energy.

The different behavior of $\mathrm{Si}$ with respect to $\mathrm{SiC}$ is due to the fact that in $\mathrm{SiC}$ the presence of two different atoms ( $\mathrm{Si}$ and $\mathrm{C}$ ) have already broken the symmetry and therefore the strain is less effective. Concerning $\mathrm{SiC}$, it is also interesting to compare with hexagonal $\mathrm{SiC}$ polytypes $(2 \mathrm{H}, 4 \mathrm{H}$, and $6 \mathrm{H})$ [32-34]. In fact, many polymorphisms of $\mathrm{SiC}$ exist which can present different and interesting electronic and optical properties with respect to the cubic SiC. For these polytypes, because of symmetry reasons, only the components $\chi_{z z z}^{(2)}, \chi_{x z x}^{(2)}$, and $\chi_{z x x}^{(2)}$ are different from zero. Comparing with the strain we found that we obtain the same order of magnitude of polytypes $\chi_{x z x}^{(2)}$ and $\chi_{z x x}^{(2)}$, while polytypes $\chi_{z z z}^{(2)}$ can induce much higher SHG response in particular in an energy region between 3 and $5 \mathrm{eV}$.

TABLE II. Calculated hydrostatic pressure (HP) and shear stress (SS) for the biaxially strained C1.8_T3.0_Y and T1.8_C3.0_Y and uniaxially strained C1.8_T3.0 and T1.8_C3.0. The upper panel is for $\mathrm{Si}$ and the lower panel is for $\mathrm{SiC}$.

\begin{tabular}{lcccc}
\hline \hline & \multicolumn{5}{c}{$\mathrm{Si}$} \\
\cline { 2 - 5 } & C1.8_T3.0 & C1.8_T3.0_Y & T1.8_C3.0 & T1.8_C3.0_Y \\
\hline HP $(\mathrm{GPa})$ & -0.34 & -0.47 & 0.59 & 0.47 \\
$\mathrm{SS}_{Y z}(\mathrm{GPa})$ & 0.00 & 0.07 & 0.00 & -0.06 \\
& \multicolumn{5}{c}{$\mathrm{SiC}$} \\
& C1.8_T3.0 & C1.8_T3.0_Y & $\mathrm{T} 1.8 \_C 3.0$ & $\mathrm{~T} 1.8 \_C 3.0 \_\mathrm{Y}$ \\
\hline $\mathrm{HP}(\mathrm{GPa})$ & -0.74 & -0.87 & 1.34 & 1.25 \\
$\mathrm{SS}_{Y z}(\mathrm{GPa})$ & 0.00 & 0.18 & 0.00 & -0.16 \\
\hline \hline
\end{tabular}



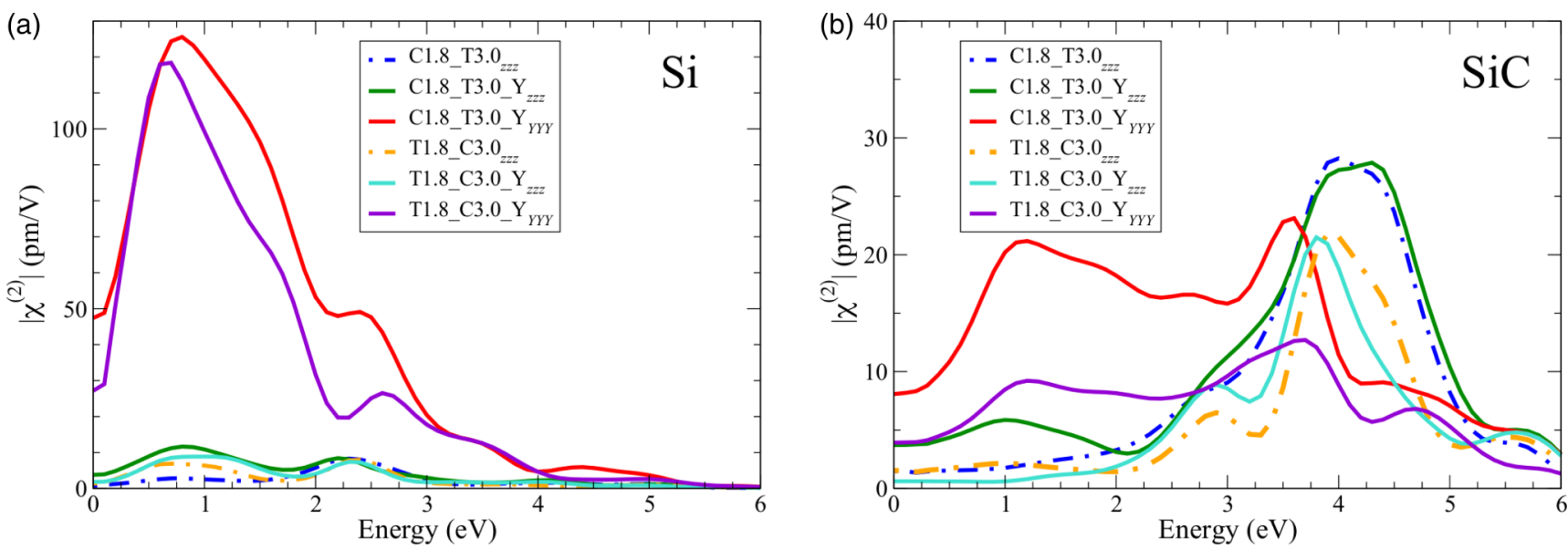

FIG. 5. (Color online) The $\chi_{z z z}^{(2)}$ and $\chi_{Y Y Y}^{(2)}$ spectra for the C1.8_X, C1.8_X_Y, T1.8_X, and T1.8_X_Y systems. Panel (a) is for Si and panel (b) is for $\mathrm{SiC}$.

\section{CONCLUSION}

We studied the correlation between the strain and the $\chi^{(2)}$ in both centrosymmetric and noncentrosymmetric materials. We considered $\mathrm{Si}$ and $\mathrm{SiC}$ as the test materials and we studied different types of strain (tensile/compressive) in different directions (uniaxial/biaxial) and for different light-polarization directions. Concerning centrosymmetric systems and uniaxial strain, we obtained that it is the mixing between compression and elongation that modulates the intensity of $\chi^{(2)}$ at different frequency regions. We found that a compressive strain can induce a huge SHG in the infrared region (telecom wavelengths), while a tensile strain mainly enhances SHG in the visible region. Furthermore, a compression will always induce stronger SHG than an elongation. This fact is of enormous importance in $\mathrm{Si}$ photonics integrated circuits and it is achievable experimentally by depositing and cladding $\mathrm{Si}$ on materials with different lattice parameters. We observed the same effect of compressive and tensile strain also in noncentrosymmetric SiC. However, it is clear that the effect of the strain is not as strong as breaking the symmetry by exploiting the different chemical nature of the atoms ( $\mathrm{Si}$ and C). Concerning the biaxial strain we found that it is more effective than uniaxial strain to enhance the $\chi^{(2)}$, in particular in the energy region around $1 \mathrm{eV}$. However, for $\mathrm{SiC}$ the biaxial strain is less effective than in Si. The different chemical nature of the atoms ( $\mathrm{Si}$ and $\mathrm{C}$ ) seemed to be much more effective than the strain in breaking the symmetry for the $\chi^{(2)}$.

The present results simulate how it is possible to tune the SHG signal in strained $\mathrm{Si}$ and $\mathrm{SiC}$. The theoretical analysis we propose has the aim to investigate and clarify the measurements of optical nonlinearity of strained silicon waveguides. In fact, the mechanism of strain enhancement of SHG is still not clear. Silicon waveguides are very complex structures and fundamental differences in the structure can induce different local $\mathrm{T}$ and/or $\mathrm{C}$ strain which can impact the SHG response dramatically. For example, Chmielak et al. [17] use the straining layer $\mathrm{Si}_{3} \mathrm{~N}_{4} / \mathrm{SiN}_{x}$ on the top and bottom of the waveguide, while Cazzanelli et al. [16] locate
$\mathrm{Si}_{3} \mathrm{~N}_{4} / \mathrm{SiN}_{x}$ just on the top. Chmielak et al. [17] show that the waveguide width strongly influences the strain and therefore the $\chi^{(2)}$. In particular, in their experimental conditions they measured the highest value of $190 \mathrm{pm} / \mathrm{V}$ by reducing the waveguide width. Instead, Cazzanelli et al. [16] found higher $\chi^{(2)}$ in larger waveguides and the highest value measured was $40 \mathrm{pm} / \mathrm{V}$.

Our model calculations can just give a qualitative description and understating of the complex process in waveguides as we basically simulate only its core. The trend we show between $\chi^{(2)}$ and uniaxial/biaxial compressive/tensile strain seems to confirm the relation between different waveguide strain profiles and the experimental $\chi^{(2)}$. The experimental energy range studied seems to indicate that different percentages of compressive strain are induced by the strain layer and we can also guess very qualitatively that in the case of the strongest $\chi^{(2)}$ the gradient of strain is mainly along one particular direction.

The design of a waveguide can permit one to control which type of strain is induced and in which direction. This can strongly optimize the geometry of the waveguides to further improve the applicability of strained waveguides for efficient devices.

\section{APPENDIX: GRAPHICAL REPRESENTATION OF PEAKS I, II, AND III ON THE C-T PLANE}

In Fig. 2 of the paper, we reported a graphical representation of the 24 uniaxial strained structures studied on a C-T plane. The points on this plane are defined by the couple $(C, T)$ and are correlated to the percentage of compression and elongation applied. For each of these structures, we also reported in Table I the magnitude of the first (I), second (II), and third (III) peaks of $\chi_{z z z}^{(2)}(\mathrm{pm} / \mathrm{V})$. The peaks are in different energy regions and in particular peak $I$ is in the infrared region around $0.5-0.7 \mathrm{eV}$, peak II is in the visible region around $2.0-2.2 \mathrm{eV}$, and peak III is still in the visible region but around 3.8-4.0 eV. The absolute and relative intensities of the peaks can be very 


\section{Peak I}

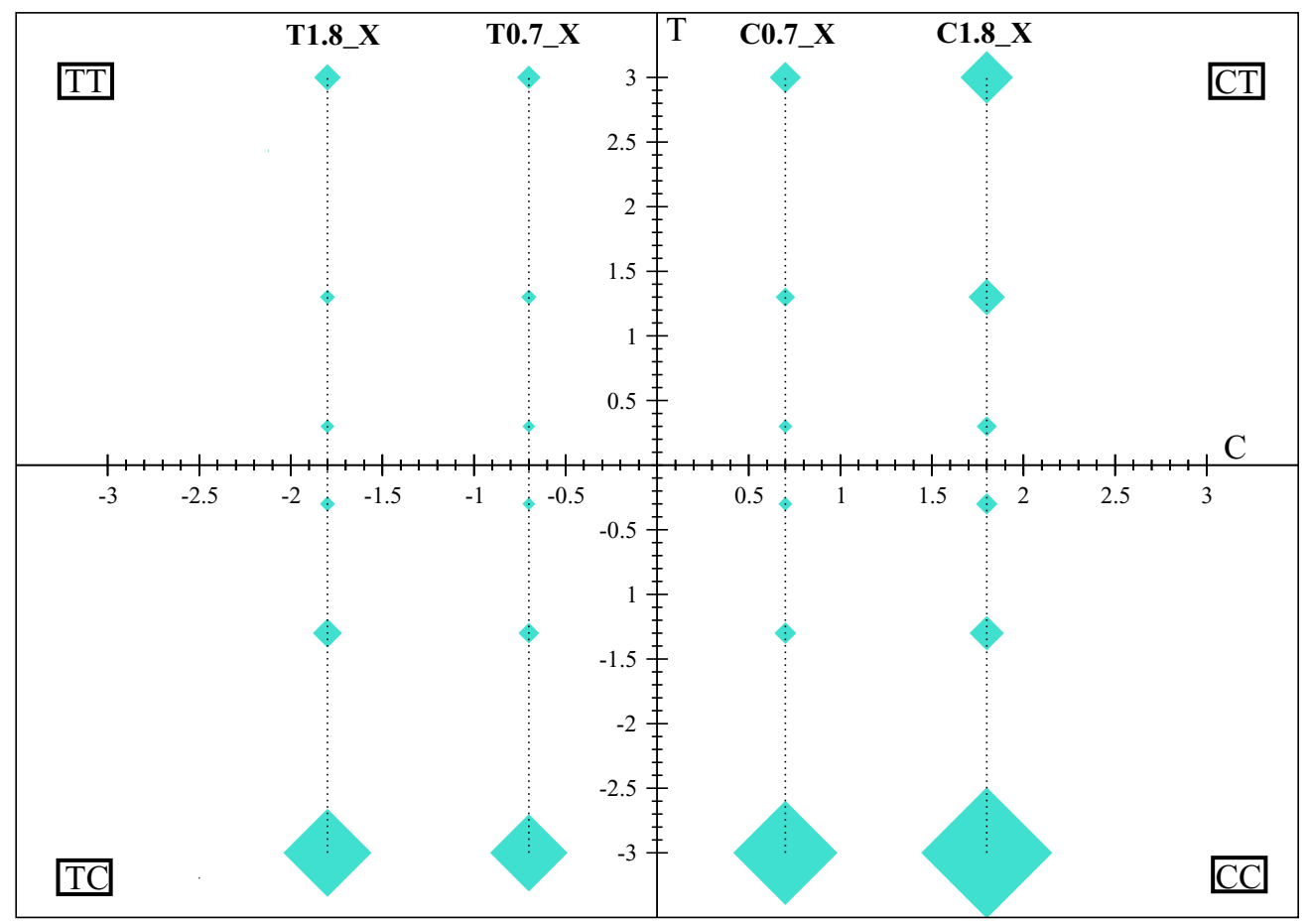

FIG. 6. (Color online) Representation of the magnitude of peak I on a C-T plane for the 24 uniaxial strained structures.

different between the spectra. The observed differences in the intensities are due to the different strain and stress between the structures.
In order to better estimate the trend between strain/stress and the $\chi_{z z z}^{(2)}$, we merged the information given in Fig. 2 and Table I of the paper and we represented the magnitude

Peak II

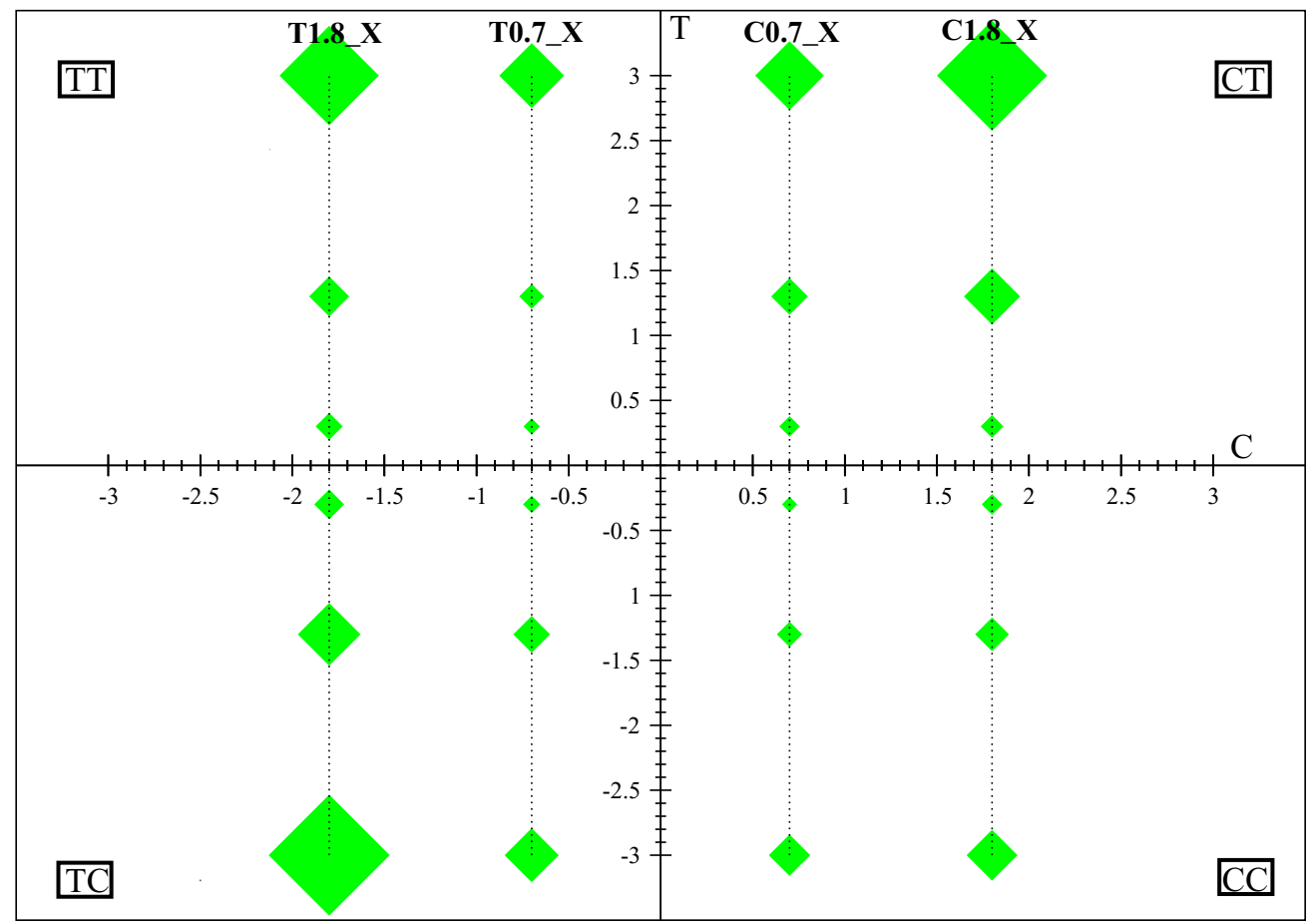

FIG. 7. (Color online) Representation of the magnitude of peak II on a C-T plane for the 24 uniaxial strained structures. 


\section{Peak III}

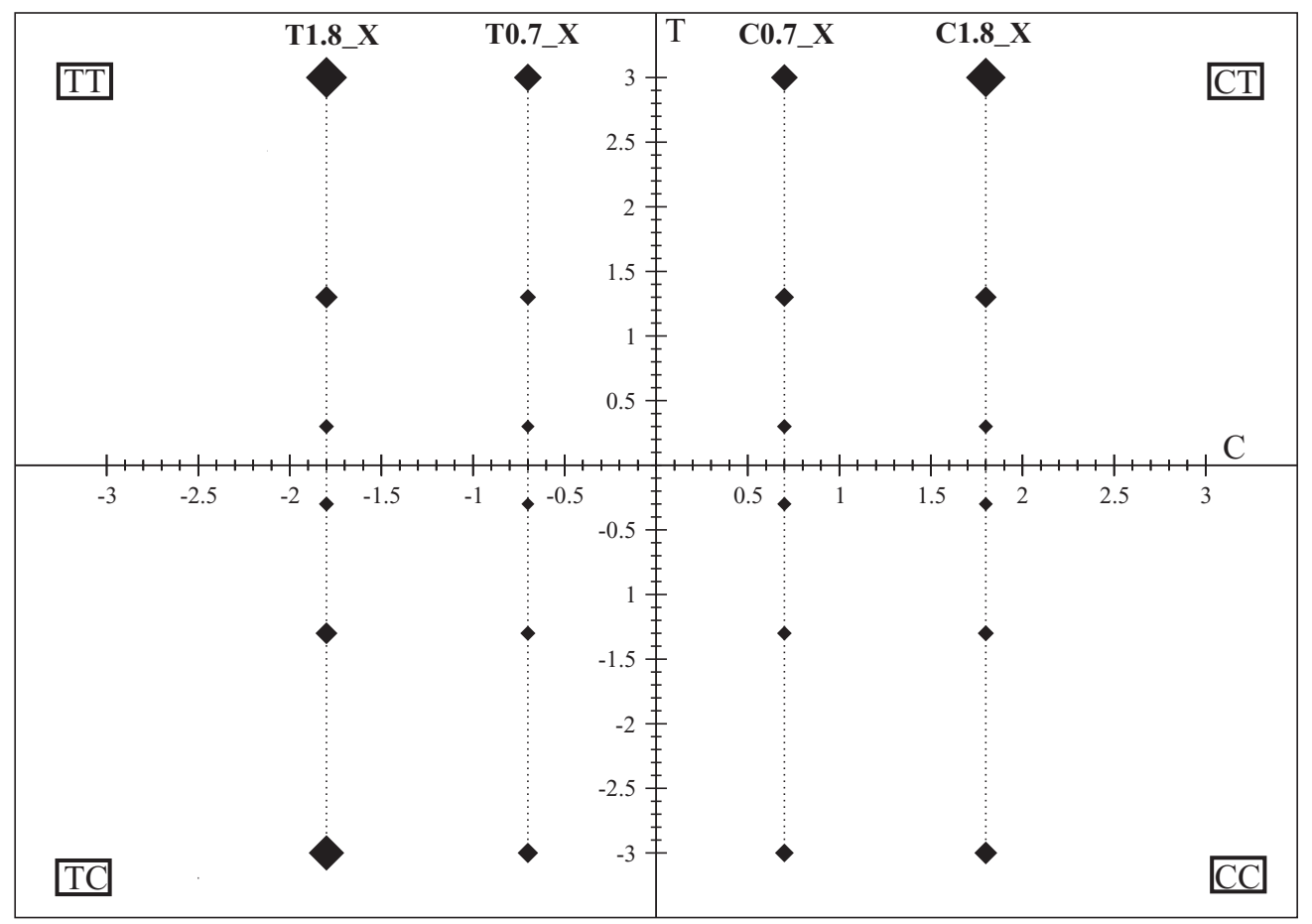

FIG. 8. Representation of the magnitude of peak III on a C-T plane for the 24 uniaxial strained structures.

of the peaks using diamonds of different size versus the representation of strain/stress as in Fig. 2 of the paper. In particular, we reported peak I in Fig. 6, peak II in Fig. 7, and finally, peak III in Fig. 8.

From Figs. 6-8 we observe that the intensity of the peaks decreases going from larger to smaller strain. In fact, for the TT and CT systems belonging to the class T1.8_X, T0.7_X, C0.7_X, and C1.8_X, the magnitude of the peaks decrease going from $\mathrm{T}=3.0 \%$ to $\mathrm{T}=1.3 \%$ and $\mathrm{T}=0.3 \%$. The same trend is observed for the TC and CC belonging to the same classes, for which the magnitude of the peaks decrease from $\mathrm{C}=3.0 \%$ to $\mathrm{C}=1.3 \%$ and $\mathrm{C}=0.3 \%$. From this graphical analysis, it is easy to observe that the strongest peak belongs to peak I, while the smallest one belongs to peak III. Moreover, it is immediately evident that a $\mathrm{C}$ strain is more efficient for the peak I and T for peaks II and III (see TT versus CC).
[1] Y. R. Shen, The Principles of Nonlinear Optics (WileyInterscience, New York, 1984).

[2] N. Bloembergen, Nonlinear Optics (Benjamin Press, New York, 1965).

[3] J. Leuthold, C. Koos, and W. Freude, Nat. Photon. 4, 535 (2010).

[4] T. Ning, O. Hyvärinen, H. Pietarinen, T. Kaplas, M. Kauranen, and G. Genty, Opt. Express 21, 2012 (2013).

[5] J. E. Mejia, B. S. Mendoza, M. Palummo, G. Onida, R. Del Sole, S. Bergfeld, and W. Daum, Phys. Rev. B 66, 195329 (2002).

[6] C. K. Chen, A. R. B. de Castro, and Y. R. Shen, Phys. Rev. Lett. 46, 145 (1981).

[7] J. Kwon, M. C. Downer, and B. S. Mendoza, Phys. Rev. B 73, 195330 (2006).

[8] P. Guyot-Sionnest, W. Chen, and Y. R. Shen, Phys. Rev. B 33, 8254 (1986).

[9] M. Bertocchi, E. Luppi, E. Degoli, V. Veniard, and S. Ossicini, J. Chem. Phys. 140, 214705 (2014).

[10] D. Hsieh, J. W. McIver, D. H. Torchinsky, D. R. Gardner, Y. S. Lee, and N. Gedik, Phys. Rev. Lett. 106, 057401 (2011).
[11] M. Galli, D. Gerace, K. Welna, T. F. Krauss, L. O'Faolain, G. Guizzetti, and L. C. Andreani, Opt. Express 18, 26613 (2010).

[12] J. Wei, A. Wirth, M. C. Downer, and B. S. Mendoza, Phys. Rev. B 84, 165316 (2011).

[13] S. V. Govorkov, V. I. Emel'Yanov, N. I. Koroteev, G. I. Petrov, I. L. Shumay, V. V. Yakovlev, and R. V. Khokhlov, J. Opt. Soc. Am. B 6, 1117 (1989).

[14] C. Schriever, C. Bohley, and R. B. Wehrspohn, Opt. Lett. 35, 273 (2010).

[15] R. S. Jacobsen, K. N. Andersen, P. I. Borel, J. Fage-Pedersen, L. H. Frandsen, O. Hansen, M. Kristensen, A. V. Lavrinenko, G. Moulin, H. Ou, C. Peucheret, B. Zsigri, and A. Bjarklev, Nature (London) 441, 199 (2006).

[16] M. Cazzanelli, F. Bianco, E. Borga, G. Pucker, M. Ghulinyan, E. Degoli, E. Luppi, V. Véniard, S. Ossicini, D. Modotto, R. P. S. Wabnitz, and L. Pavesi, Nat. Mater. 11, 148 (2012).

[17] B. Chmielak, C. Matheisen, C. Ripperda, J. Bolten, T. Wahlbrink, M. Waldow, H. Kurz, B. Ménaert, J. Zaccaro, and G. Aka, Opt. Express 21, 25324 (2013). 
[18] C. Schriever, F. Bianco, M. Cazzanelli, M. Ghulinyan, C. Eisenschmidt, J. de Boor, A. Schmid, J. Heitmann, L. Pavesi, and J. Schilling, Adv. Opt. Mater. 3, 129 (2015).

[19] C. Schriever and R. B. Wehrspohn, Nat. Mater. 11, 96 (2012).

[20] F. Bianco, K. Fedus, F. Enrichi, R. Pierobon, M. Cazzanelli, M. Ghulinyan, G. Pucker, and L. Pavesi, Semicond. Sci. Technol. 27, 085009 (2012).

[21] P. Damas, X. Le Roux, D. Le Bourdais, E. Cassan, D. MarrisMorini, N. Izard, T. Maroutian, P. Lecoeur, and L. Vivien, Opt. Express 22, 22095 (2014).

[22] K. Saito, T. Tanabe, and Y. Oyama, Opt. Express 22, 16660 (2014).

[23] M. W. Puckett, J. S. T. Smalley, M. Abashin, A. Grieco, and Y. Fainman, Opt. Lett. 39, 1693 (2014).

[24] E. Luppi, H.-C. Weissker, S. Bottaro, F. Sottile, V. Veniard, L. Reining, and G. Onida, Phys. Rev. B 78, 245124 (2008).

[25] X. Gonze, B. Amadon, P.-M. Anglade, J.-M. Beuken, F. Bottin, P. Boulanger, F. Bruneval, D. Caliste, R. Caracas, M. Cote, T. Deutsch, L. Genovese, Ph. Ghosez, M. Giantomassi, S. Goedecker, D. R. Hamann, P. Hermet, F. Jollet, G. Jomard, S. Leroux et al., Comput. Phys. Commun. 180, 2582 (2009).
[26] X. Gonze, G.-M. Rignanese, M. Verstraete, J.-M. Beuken, Y. Pouillon, R. Caracas, F. Jollet, M. Torrent, G. Zerah, M. Mikami, Ph. Ghosez, M. Veithen, J.-Y. Raty, V. Olevano, F. Bruneval, L. Reining, R. Godby, G. Onida, D. R. Hamann, and D. C. Allan, Z. Kristallogr. 220, 558 (2005).

[27] O. H. Nielsen and R. M. Martin, Phys. Rev. B 32, 3792 (1985).

[28] E. Luppi, H. Hübener, and V. Véniard, Phys. Rev. B 82, 235201 (2010).

[29] S. Botti, F. Sottile, N. Vast, V. Olevano, L. Reining, H.-C. Weissker, A. Rubio, G. Onida, R. D. Sole, and R. W. Godby, Phys. Rev. B 69, 155112 (2004).

[30] R. W. Godby, M. Schlüter, and L. J. Sham, Phys. Rev. B 35, 4170 (1987).

[31] E. Luppi, H. Hübener, and V. Véniard, J. Chem. Phys. 132, 241104 (2010).

[32] H. Hübener, E. Luppi, and V. Véniard, Phys. Rev. B 83, 115205 (2011).

[33] S. N. Rashkeev, W. R. L. Lambrecht, and B. Segall, Phys. Rev. B 57, 9705 (1998).

[34] J. Chen, Z. H. Levine, and J. W. Wilkins, Phys. Rev. B 50, 11514 (1994). 